\title{
SPACES OF EQUIVARIANT SELF-EQUIVALENCES OF SPHERES
}

\author{
BY J. C. BECKER ${ }^{1}$ AND R. E. SCHULTZ ${ }^{2}$
}

Communicated by William Browder, July 13, 1972

\begin{abstract}
Let $F\left(S^{m}\right)$ denote the identity component of the space of homotopy self-equivalences of $S^{m}$ and let $F=\operatorname{inj} \lim _{m} F\left(S^{m}\right)$. This paper studies the homotopy properties of certain equivariant analogs of the infinite loop space $F$.
\end{abstract}

1. Introduction. Let $G$ be a compact Lie group and let $W$ be a free, finite dimensional, real $G$-module equipped with a $G$-invariant metric. Let $S(W)$ be the unit sphere of $W$ and denote by $F(W)$ the identity component of the space of equivariant self-equivalences of $S(W)$ with the compact-open topology.

If $V$ and $W$ are free $G$-modules as above, then $V \oplus W$ is also a free $G$-module. Since $S(V \oplus W)$ is equivariantly homeomorphic to the join of $S(V)$ and $S(W)$, there is a continuous inclusion of $F(V)$ into $F(V \oplus W)$ defined by taking joins with the identity on $S(W)$. In particular, if $k W$ denotes the direct sum of $k$ copies of $W$, there is an inclusion of $F(k W)$ in $F((k+1) W)$. Define

$$
F_{G}=\operatorname{inj} \lim _{k} F(k W) .
$$

If $G$ is the trivial group then $F_{G}=F$ is a familiar and widely studied object. An important aspect of this space is the existence of two infinite loop space structures, one induced by composition multiplication, the other induced by a canonical homotopy equivalence from $F$ to the identity component of inj $\lim _{m} \Omega^{m}\left(S^{m}\right)$. One can show that $F_{G}$ also has an infinite loop space structure induced by composition multiplication. Our results generalize to $F_{G}$ the second infinite loop space structure on $F$.

Let $B G$ denote a classifying space for $G$, let $\mathfrak{g}$ be the Lie algebra of $G$ and let $G$ act on $\mathfrak{g}$ via the adjoint representation. The balanced product of $E G$ and $\mathfrak{g}$ is a vector bundle over $B G$ that we shall call $\zeta$. Let $B G^{\zeta}$ denote its Thom space.

THEOREM 1. On the category of connected finite $\mathrm{CW}$-complexes there is a natural equivalence of homotopy functors

AMS (MOS) subject classifications (1970). Primary 55D10, 55D35; Secondary 55E45.

Key words and phrases. Free linear representation, equivariant self-equivalence, infinite loop space, transfer map, ex-space.

${ }^{1}$ Partially supported by NSF Grant GP-34197.

2 Partially supported by NSF Grant GP-19530. 


$$
\lambda_{G}:\left[; F_{G}\right] \rightarrow\left\{; B G^{\zeta}\right\}
$$

Here $\{A ; B\}$ denotes the homotopy classes of pointed $S$-maps from $A$ to $B$.

If $Y$ is a pointed space, let $Q_{0}(Y)$ be the identity component of inj $\lim _{k} \Omega^{k} S^{k}(Y)$. The exponential law provides a natural equivalence from $\{; Y\}$ to $\left[; Q_{0}(Y)\right]$ on the category of connected finite $\mathrm{CW}$-complexes. Combining this with Theorem 1 , we obtain the following result.

THEOREM 2. The space $F_{G}$ is homotopy equivalent to $Q_{0}\left(B G^{\zeta}\right)$.

If $G$ is the trivial group, Theorem 1 reduces to the usual equivalence

$$
\lambda[; F] \rightarrow\left\{; S^{0}\right\}
$$

given by sending $f: X \rightarrow F\left(S^{n}\right)$ to the map $h(f): X * S^{n} \rightarrow S^{n+1}$ obtained by applying the Hopf construction to the adjoint of $f$. If $G$ is a finite group, then $\zeta$ is 0-dimensional and $B G^{\zeta}=B G^{+}$, the disjoint union of $B G$ with a point. In this case $F_{G}$ has the homotopy type of $Q_{0}(B G) \times Q_{0}\left(S^{0}\right)$. The only compact Lie groups of positive dimension that act freely on spheres are $S^{1}, S^{3}$, and $N\left(S^{1}\right)$, the normalizer of $S^{1}$ in $S^{3}$ [5]. If $G=S^{1}$ then $\zeta$ is a trivial line bundle and in this case $F_{G}$ has the homotopy type of $Q_{0}\left(C P^{\infty}\right)$ $\times Q_{0}\left(S^{1}\right)$. If $G=S^{3}$, then $B G^{\zeta}$ is an infinite dimensional quasi-projective space as defined by James (see [2, Proposition (5.3)]).

2. Naturality properties. Let $G$ be as above and let $H$ be a closed subgroup of $G$. Then we may take $B H$ to be $E G / H$, and the canonical map from $B H$ to $B G$ to be the projection. Techniques of J. M. Boardman [4] imply the existence of a "wrong way" map (in the stable homotopy category)

$$
\tau: B G^{\zeta(G)} \rightarrow B H^{\zeta(H)} .
$$

If $G$ and $H$ are finite, $\tau$ agrees with the transfer defined in [9]. Let

$$
\rho: F_{G} \rightarrow F_{H}
$$

denote the natural forgetful map.

THEOREM 3. The following diagram is commutative

$$
\begin{aligned}
& {\left[; F_{G}\right] \stackrel{\rho_{*}}{\longrightarrow}\left[; F_{H}\right]} \\
& \downarrow \lambda_{G} \quad \downarrow \lambda_{H} \\
& \left\{\quad ; B G^{\zeta(G)}\right\} \stackrel{\tau_{*}}{\rightarrow}\left\{\quad ; B H^{\zeta(H)}\right\} .
\end{aligned}
$$

If $G$ and $H$ are finite, the map

\footnotetext{
${ }^{3}$ ADDED IN PROOF. Spaces related to $F_{G}$ have been studied by G. Segal [12] using bordism techniques. Theorem 2 is similar to Proposition 4 of [12].
} 


$$
p_{*}^{+}:\left\{; B H^{+}\right\} \rightarrow\left\{; B G^{+}\right\}
$$

has a geometrical interpretation in terms of a transfer map $t: F_{H} \rightarrow F_{G}$; details will appear elsewhere.

3. Applications. The above results are useful in describing the image of

$$
\rho_{*}: \pi_{*}\left(F_{G}\right) \rightarrow \pi_{*}\left(F_{H}\right) .
$$

For example, a theorem of D. S. Kahn and S. B. Priddy [8] implies that the transfer

$$
\tau: \Sigma_{n}\left(R P^{\infty+}\right) \rightarrow \Sigma_{n}\left(S^{0}\right), \quad n>0,
$$

is surjective. Hence we have the following.

THEOREM 4. The forgetful map $\rho_{*}: \pi_{*}\left(F_{Z_{2}}\right) \rightarrow \pi_{*}(F)$ is surjective.

On the other hand we have the following result.

THEOREM 5. Let $k$ be a positive integer, let $\sigma_{k} \in \pi_{8 k-1}(F)$ generate the image of $J$, and let $\mu_{k} \in \pi_{8 k+1}(F)$ be an Adams-Barratt element [1]. Then neither $\sigma_{k}$ nor $\mu_{k}$ is in the image of $\rho_{*}: \pi_{*}\left(F_{S^{1}}\right) \rightarrow \pi_{*}(F)$.

Geometrical applications of the result on $\mu_{k}$ will be given in [10].

4. Spaces over $B$. Fix a $C W$-complex $B$ and let $\mathscr{C}(B)$ denote the category having objects $\xi=\left(E_{\xi}, B, p_{\xi}, \Delta_{\xi}\right)$ where $p_{\xi}: E_{\xi} \rightarrow B$ is a fiber bundle and $\Delta_{\xi}$ is a cross section to $p_{\xi}$. We assume that $\xi$ is admissible in the sense of [3]. In the terminology of James [7], $\xi$ is an ex-space of $B$. The set $\left[\xi, \xi^{\prime}\right]$ of maps in $\mathscr{C}(B)$ is the set of homotopy classes of fiber and cross section preserving maps $E_{\xi} \rightarrow E_{\xi^{\prime}}$. The category $\mathscr{C}(B)$ is a natural extension of the category of pointed spaces, and much of the homotopy theory of pointed spaces can be extended to $\mathscr{C}(B)$. For detailed accounts see [3], [6], [7].

Let $\xi \wedge \alpha$ denote the fiberwise reduced join of $\xi$ and $\alpha$ and define

$$
\sigma:\left[\xi ; \xi^{\prime}\right] \rightarrow\left[\xi \wedge \alpha ; \xi^{\prime} \wedge \alpha\right]
$$

by $f \rightarrow f \wedge 1$. We then have the following suspension theorem (compare [6, Theorem (7.4)]).

THEOREM 6. Assume that $\alpha$ is a sphere bundle and the fiber of $\xi^{\prime}$ is $(n-1)$ connected. Then $\sigma$ is injective if $E_{\xi}$ is $(2 n-1)$-coconnected and surjective if $E_{\xi}$ is $2 n$-coconnected.

Let $T(\xi)=E_{\xi} / \Delta_{\xi}(B)$. If $X$ is a space with base point $x_{0}$ let $\dot{X}$ denote the object $(B \times X, B, p, \Delta)$ where $p(b, x)=b$ and $\Delta(b)=(b, x)$. Note that $T(X \wedge \xi)=X \wedge T(\xi)$. Observe also that the projection map $B \times X$ 
$\rightarrow X$ induces a one-one correspondence $[\xi ; X] \rightarrow[T(\xi) ; X]$.

If $\beta$ is a vector bundle over $B$ let $\bar{\beta}$ denote the object of $\mathscr{C}(B)$ obtained by taking the fiberwise one point compactification of $E_{\beta}$ and letting $\Delta_{\bar{\beta}}$ be the cross section at infinity.

5. Proof of Theorem 1. Let $W$ be a free $G$-module of dimension $n$, let $M(W)=S(W) / G$ and let $\xi=(S(W) \times S(W) / G, M(W), p, \Delta)$ where $p\left[w, w^{\prime}\right]$ $=[w]$ and $\Delta[w]=[w, w]$. Suppose that $X$ is a finite connected complex and $\operatorname{dim}(X)<n-2$. We have a bijection

$$
\theta:[X ; F(W)] \rightarrow[\dot{X} ; \xi]
$$

defined as follows: given $f: X \rightarrow F(W)$ define

by

$$
\theta(f): M(W) \times X \rightarrow S(W) \times S(W) / G
$$

$$
\theta(f)([w], x)=[w, f(x)(w)] .
$$

If $M$ is a smooth manifold let $\tau(M)$ denote its tangent bundle. Let $\zeta$ denote the bundle with fiber $g$ associated with the principal bundle $S(W)$ $\rightarrow M(W)$. We then have $[11]$

$$
\xi \simeq \overline{\tau(S(W)) / G} \simeq \overline{\tau(M(W)) \oplus \zeta}
$$

Making this identification (and abbreviating $\tau(M(W))$ to $\tau)$ we have

$$
\theta:[X ; F(W)] \rightarrow[\dot{X} ; \overline{\tau \oplus \zeta}]
$$

Now choose (a) an embedding $h: M(W) \subset R^{s}$ and (b) a monomorphism $\phi: \zeta \rightarrow B \times R^{t}$. Let $v$ denote the normal bundle determined by $h$ and $\zeta^{\prime}$ the complementary bundle determined by $\phi$. From this data we obtain $\left(\mathrm{a}^{\prime}\right)$ an equivalence $\psi: \overline{(\tau \oplus \zeta) \oplus\left(v \oplus \zeta^{\prime}\right)} \rightarrow S^{s+t}$ and $\left(\mathrm{b}^{\prime}\right)$ a duality map $\mu: S^{s+t} \rightarrow T(\zeta) \wedge T\left(v \oplus \zeta^{\prime}\right)$.

Define

$$
\kappa:[\dot{X} ; \overline{\tau \oplus \zeta}] \rightarrow\left[X \wedge T\left(v \oplus \zeta^{\prime}\right) ; S^{s+t}\right]
$$

to be composition

$$
\begin{aligned}
{[\dot{X} ; \overline{\tau \oplus \zeta}] \stackrel{\sigma}{\rightarrow}\left[\dot{X} \wedge \overline{v \oplus \zeta^{\prime}} ; \tau \overline{\oplus \zeta \oplus v \oplus \zeta^{\prime}}\right] } \\
\stackrel{\psi_{*}}{\rightarrow}\left[\dot{X} \wedge \overline{v \oplus \zeta^{\prime}} ; \dot{S}^{s+t}\right] \rightarrow\left[X \wedge T\left(v \oplus \zeta^{\prime}\right) ; S^{s+t}\right] .
\end{aligned}
$$

Since $\operatorname{dim}(X)<n-2, \sigma$ and hence $\kappa$ is bijective.

The duality map $\mu$ defines a bijection

$$
D_{\mu}:\left\{X \wedge T\left(v \oplus \zeta^{\prime}\right) ; S^{s+t}\right\} \rightarrow\{X ; T(\zeta)\}
$$


Since we are in the stable range we may define

$$
\lambda_{W}:[X, F(W)] \rightarrow\{X ; T(\zeta)\}
$$

by $\lambda_{W}=D_{\mu} \kappa \theta$. It is easily seen that $\lambda_{W}$ is independent of the choice of $h$ and $\phi$. Moreover, if $V$ is a second free $G$-module, it is compatible with the inclusion $F(V) \rightarrow F(V \oplus W)$ in the obvious sense. Now $\lambda_{G}$ in Theorem 1 is defined to be inj $\lim _{k} \lambda_{k W}$.

\section{REFERENCES}

1. J. F. Adams, On the groups $J(X)$. IV, Topology 5 (1966), 21-71. MR 33 \# 6628.

2. M. F. Atiyah, Thom complexes, Proc. London Math. Soc. (3) 11 (1961), 291-310. MR 24 \# A1727.

3. J. C. Becker, On the existence of $A_{k}$-structures on stable vector bundles, Topology 9 (1970), 367-384. MR 42 \# 1135.

4. J. M. Boardman, Stable homotopy theory, University of Warwick, 1966 (mimeographed notes).

5. G. E. Bredon, Introduction to compact transformation groups, Academic Press, New York, 1972.

6. I. M. James, Bundles with special structure. I, Ann. of Math. (2) 89 (1969), 359-390. MR 39 \# 4868.

7. $\longrightarrow$ Ex-homotopy theory. I, Illinois J. Math. 15 (1971), 324-337.

8. D. S. Kahn and S. B. Priddy, Applications of the transfer to stable homotopy theory (to appear).

9. F. W. Roush, Transfer in generalized cohomology theories, Thesis, Princeton University, Princeton, N.J.

10. R. E. Schultz, Homotopy sphere pairs admitting semifree differentiable actions (to appear).

11. R. H. Szczarba, On tangent bundles of fibre spaces and quotient spaces, Amer. J. Math. 86 (1964), 685-697. MR 30 \#2522.

12. G. B. Segal, Equivariant stable homotopy theory, Proc. Internat. Congress Math. (Nice, 1970), vol. 2, Gauthier-Villars, Paris, 1971, pp. 59-64.

Department of Mathematics, Purdue University, Lafayette, Indiana 47907 